\title{
Colombia en la Antártida: aproximaciones a una investigación científico/social... un llamado a la academia/estado/sociedad
}

\section{Colombia in the Antarctica: Approaches to a scientific/social investigation... A call to the academy/state/society}

\author{
William Mauricio Lopera Ramírez ${ }^{1}$ \\ Escuela Superior de Guerra, Colombia \\ "El océano, el gran unificador, es la única \\ esperanza del hombre, ahora como nunca, la \\ antigua frase tiene un nuevo significado: todos \\ estamos en el mismo bote" (Cousteau, 1992).
}

\section{RESUMEN}

La Antártida, para muchos desconocida, para otros épica, y para las grandes potencias del globo un lugar de posicionamiento geoestratégico sin precedentes, merece la atención no solo del andamiaje estatal como tal, sino de una triada donde a este se anejan la academia y la sociedad desde su expresión

1 Capitán de Fragata Armada Nacional de la República de Colombia, profesional en Relaciones Internacionales y Estudios Políticos, Especialista en Política y Estratégica Marítima, Especialista en Defensa y Seguridad del Estado, Magister en Intercultural Conflict Management. Mauricio lopera ramirez maurilopra11@gmail.com Código ORCID: 0000-0002-9696-9347

visionaria/participativa que permite entregar el valor necesario a un "proyecto país" con enfoque diferencial, direccionado a los intereses nacionales, desde el entendido que estos trascienden más allá de las fronteras, donde las relaciones internacionales y la política exterior de un estado permiten la gestión.

Con el fin de adentrarnos en el objeto de estudio, es preciso contextualizar algunos aspectos que permitirán un recorrido entre la relevancia y la fastuosa historia del Continente Blanco. En principio, se hará referencia a la importancia estratégica, sus características y lo que sígnica 
para el hemisferio en conjunto con el majestuoso Océano Antártico que lo rodea.

\section{ABSTRAC}

Antarctica, for many unknown, for others epic, and for the great powers of the globe a place of unprecedented geostrategic positioning that deserves the attention not only of the state scaffolding as such, but of a triad where academia and the society from its visionary I participatory expression that allows to deliver the necessary value to a "project", with a differential approach directed at national interests, from the understanding that these transcend beyond borders, where international relations and the foreign policy of a state allow management.

In order to delve into the object of study, it is necessary to contextualize some aspects that will allow a journey between the relevance and the lavish history of the White Continent. In principle, reference will be made to the strategic importance, its characteristics and what it means for the hemisphere in conjunction with the majestic Antarctic Ocean that surrounds it.

KEYWORDS: Antarctica, country project, differential approach

\section{LARELEVANCIADE LAANTÁRTICA}

De acuerdo con el Diccionario Panhispánico de Dudas (2005), es válido utilizar cualquiera de los dos términos: Antártida o Antártica, que hacen referencia al adjetivo latino antarcticus que significa "opuesto al Ártico" (Corin, 2018)

El término "Antártida" describe el conjunto de tierras situadas en el Polo Sur terrestre. Este término es más usado en España y en la mayor parte de América, debido a la analogía de la terminación \{-da\} de otros topónimos como Holanda, Nueva Zelanda y Atlántida, entre otros. Se suele denominar indistintamente Antártida o Antártica, aunque se prefiere el primer término, ya que es el más usado por cartógrafos y geógrafos. La denominación de "Antártica" se da porque el territorio más alejado y desconocido del planeta está rodeado por el océano Antártico (Bordese, 2008).

No cabe duda de que, de todos los continentes, la Antártida es el que más peculiaridades presenta, no sólo por ser el más frio y seco de todos, sino porque el $99 \%$ de su territorio está cubierto con hielo. Además, esta región fue la última en ser descubierta, es la más deshabitada e inexplorada de todas, y también es el continente más pacífico, pues nunca se ha registrado una guerra, a pesar de ser reclamado por siete países (Armada española, 2019). Algo muy particular e incluso contradictorio de la Antártida es que "[l]a historia de un continente comienza cuando llega el hombre y la presencia humana es muy reciente en la Antártica" (Centro cultural argentino de montaña, 2009). De hecho, "(...) es el único del planeta que no cuenta con una población humana nativa" (Europa Press, 2017).

Este continente cuenta con una extensión de 14 millones de kilómetros cuadraros, es decir, prácticamente 12 veces el tamaño de Colombia. Sumado a esto, es un grupo de islas unidas entre sí por numerosas capas de hielo seculares en torno al Polo Sur y el $99 \%$ de su territorio está cubierto por grandes masas glaciares que alcanzan un volumen de 30.000 millones de kilómetros cúbicos de hielo. Como si fuera poco, cuenta con la mayor reserva de agua dulce del planeta, con $77 \%$ del total mundial y $90 \%$ del hielo terrestre. También, contiene un registro único de lo que fue el clima en nuestro planeta hace varios miles de años. Todas estas características hacen de la Antártida un continente clave para entender cómo funciona el planeta al ser considerado el "laboratorio natural" más grande del mundo porque es el sitio donde es posible visualizar, de primera 
mano, los efectos del impacto humano en el medio ambiente (Rowlatt, 2020).

Este territorio también es de gran importancia para la vida de la tierra por la influencia que ejerce sobre muchos procesos naturales, ya que contiene la mayor cantidad de hielo del planeta y es el motor que regula la atmósfera terrestre y su equilibrio global (Green Peace, 2020). Además, tiene influencia directa sobre el clima de la tierra y sobre sus habitantes, $y$ es un termostato que regula el clima del planeta. El doctor Bolívar Cáceres, responsable del Programa Glaciar del Instituto Nacional de Meteorología de Ecuador, indica que "[t]odos los océanos están conectados, y por eso cualquier cosa que ocurra en este continente puede dar lugar a una sequía intensa o a unas lluvias torrenciales en distintos puntos del planeta. Es como un efecto mariposa" (Bolívar, citado por Talarn, 2017).

Finalmente, la temperatura media anual de la Antártida oscila entre los $-10{ }^{\circ} \mathrm{C}$ en la costa Antártica y $-60{ }^{\circ} \mathrm{C}$ en las partes más elevadas del interior. En febrero de 2020, esta zona alcanzó una temperatura récord de 18.3 ${ }^{\circ} \mathrm{C}$, algo que "(...) no se había presentado ni siquiera en la temporada de verano" (Rowlatt, 2020). Además, el 10 de agosto de 2010, se registró la temperatura más baja de $-93.2{ }^{\circ} \mathrm{C}$ en la meseta Antártica, superando los $-89^{\circ} \mathrm{C}$ registrados en 1989.

\section{EL OCÉANO ANTÁRTICO}

El Océano Austral es uno de los ecosistemas marinos menos alterados $\mathrm{y}$, a su vez, uno de los más productivos del mundo, con una gran cantidad de especies que no se encuentran en ningún otro lugar. Desde 1980, existe la Convención sobre la Conservación de los Recursos Vivos Marinos Antárticos (CCRVMA) cuyo objetivo es encontrar un equilibrio entre la conservación y la explotación racional de los recursos vivos (Comisión para la Conservación de los Recursos Vivos Marinos Antárticos, 2019). Como su nombre lo indica, el océano Antártico es el que queda más al sur del planeta y se encuentra prácticamente rodeando al continente antártico. Se considera que este océano empieza a partir de latitud $60^{\circ}$ sur y recorre $360^{\circ}$, lo que lo hace un círculo completo siguiendo las líneas imaginarias alrededor de la tierra (GeoEnciclopedia, s.f.).

Este océano es relativamente nuevo en los mapas, ya que anteriormente los tres grandes océanos llegaban hasta la Antártida, pero, tras un largo proceso, la Organización Hidrológica Internacional, en el 2000, logró que sus miembros reconocieran este océano separado y empezaran a plasmarlo en sus documentos a partir del 2002 (GeoEnciclopedia, s.f.). De los cinco océanos (Pacífico, Atlántico, Índico, Antártico y Ártico), el Antártico es el cuarto en cuanto a tamaño, siendo solamente mayor que el Océano Ártico. La línea costera de este océano es de 17,968 km y su superficie es de 20,327 km² (GeoEnciclopedia, s.f.). Además, la temperatura de este océano, que rodea al Polo Sur, es extremadamente baja oscilando entre los $-2{ }^{\circ} \mathrm{C}$ y los $-10^{\circ} \mathrm{C}$ en las épocas más calientes (Green Peace, 2020).

Aunado a lo anterior, "[a]lgunos científicos consideran que este océano debe ser limitado por la Convergencia Antártica, una zona de delimitación natural donde las frías aguas del sur que fluyen hacia el norte, se encuentran con aguas más calientes de los océanos Pacífico, Atlántico e Índico según la zona y por tener menor densidad se hunden debajo de ellas" (GeoEnciclopedia, s.f.). La convergencia Antártica, al ser una delimitación natural, tiene la gran desventaja que varía según la época del año (GeoEnciclopedia, s.f.). 


\section{LA ANTÁRTICA Y SU HISTORIA}

Desde el inicio de la humanidad, este territorio permaneció inexplorado por miles de años. A pesar de esto, filósofos griegos se cuestionaban sobre la existencia de una terra australis ignota (tierra desconocida del sur), un continente imaginario con orígenes en la Grecia clásica que solía aparecer en los mapas europeos a partir del siglo $X V$ y hasta el siglo $X V I I I$, pero que ningún ser humano había avistado aún (Alberini, 1971). El 24 de enero de 1539, Pedro Sánchez de la Hoz firmó con el emperador Carlos V una capitulación por la que fue nombrado gobernador de las tierras al sur del estrecho de Magallanes, llamadas entonces Terra Australis, y de todas las islas no asignadas que descubriese; sin embargo, esta capitulación no tuvo efecto alguno (Céspedes, 2020).

La Antártica había sido avistada en el año 1603 por el navegante español Gabriel de Castilla; lastimosamente su contribución al conocimiento geográfico fue ignorada por mucho tiempo. Como dato curioso, en 1989 fue bautizada una base científica española en la Antártida en su honor (Pinedo, National Geographic, 2018). Siglo y medio después, el siguiente en aproximarse al gélido continente fue el británico James Cook, quien llegó a cruzar el círculo polar antártico en 1773, aunque sin avistar tierra firme, quedándose a $120 \mathrm{~km}$ de la costa (Gabarró i Raurich, 2013). Luego, se han registrado las siguientes expediciones

Los siguientes en acercarse a la Antártida fueron Fabian Gottieb y Mijaíl Lázarev al mando de una expedición rusa para la circunnavegación del globo en 1820, llegando a $32 \mathrm{~km}$ de la Tierra de la Reina Maud. Esta última ocurrió tres días antes de la expedición irlandés Edward Brand para la corona británica, por lo que usualmente se les considera a ambos como codescubridores de la Antártida durante 1820. (Gabarró i Raurich, 2013)
Esta última, ocurrió tres días antes de la expedición irlandés Edward Brand para la Corona Británica, por lo que usualmente se les considera a ambos como codescubridores de la Antártida durante 1820. Así mismo, el cazador de ballenas estadounidense Nataniel Palmer, llegó a las Islas Orcadas diez meses después, por lo que algunos también lo incluyen en el grupo de los descubridores. Durante el resto del siglo, se continuaron haciendo exploraciones del continente, aunque sin ningún asentamiento permanente por las hostiles condiciones de vida.

A partir del siglo $X X$, se insertan en la carrera por la Antártica nuevos expedicionarios como el británico Robert Falcon Scott, en su expedición denominada Discovery, la cual se diera en el primer quinquenio del siglo. Se obtuvieron sendos hallazgos en científicos, especialmente, para la meteorología y el magnetismo, entre otros. Esta expedición despertó aún más el interés antártico en el continente europeo (Rowlatt, BBC News, 2020).

Paralelamente, los suecos perseguían el mismo propósito, pero la que sería su primera expedición también quedaría registrada en la historia como el primer suceso desafortunado en la Antártica, debido a que el buque en que viajaban científicos e investigadores, incluso originarios de Argentina, se averió por la adversidad del Continente Blanco. La tripulación sobrevivió y fue rescatada con vida después de haber sorteado las adversidades climáticas de este territorio inexplorado (Gabarró i Raurich, 2013). En el segundo decenio del siglo XX, Noruega lanzó su gran expedición Antártica, dirigida por Roald Amundsen y remembrada en la historia por ser la primera en arribar al Polo Sur Geográfico en la latitud 90 grados sur (Estirado, El Periódico, 2016).

Después de un siglo, los exploradores abolieron su espíritu aventurero para involucrar en absoluto la ciencia. En la actualidad Colombia, aspira formar parte de esta historia, ya el país 
ha demostrado su intención y su capacidad. La persistencia, la voluntad política y los alcances de la diplomacia, sin duda le otorgaran ese privilegio.

\section{LAS RECLAMACIONES}

¿Por qué Reino Unido, Francia, Noruega, Australia, Nueva Zelanda, Chile y Argentina han trazado fronteras en el mapa de la Antártica y se han repartido el vacío de hielo con reivindicaciones territoriales? ¿Existen intereses económicos? ¿Esto se debe a una posición estratégica? Se dice que los gobiernos realmente quieren saber lo que hay debajo del hielo y existe una palabra en el ambiente: petróleo. Algunas predicciones indican que la cantidad de petróleo en la Antártida podría ser de 200.000 millones de barriles, mucho más que Kuwait o Abu Dhabi (Rowlatt, 2020). Adicionalmente, los cielos de Antártida son inusualmente claros y libres de interferencias de radio, por lo que son ideales para la investigación del espacio profundo y el seguimiento por satélite. Sumado a esto, son ideales para el establecimiento de redes de vigilancia encubierta y el control remoto de sistemas de armas ofensivas (Rowlatt, 2020). Estas pueden ser algunas de las razones por las cuales es un área tan apetecida.

A partir del siglo $X X$, comenzaron las reclamaciones territoriales de la Antártida, específicamente a partir de 1940 (Gabarró i Raurich, 2013). El primero fue el Reino Unido, seguido por Chile y Argentina. El Reino Unido lanzó la Operación Tabarín en la que estableció bases permanentes ocupadas en 1943 (Gabarró i Raurich, 2013). El Reino Unido inicia sus reclamaciones aduciendo haber liderado varias expediciones. A esta intención se suman Australia y Nueva Zelanda, y Francia aprovecha su posición en algunas islas adyacentes para, de igual manera, iniciar reclamaciones antárticas. Noruega utilizo similar argumento sobre las expediciones lideradas para manifestar su interés de reclamación en 1939 (Rowlatt, BBC News, 2020).

Durante el último decenio de la primera mitad del siglo XX, se presentaron controversias por la convergencia de intereses en las reclamaciones de territorio antártico. Por nombrar alguna, Chile $y$ el Reino Unido protagonizaron un incidente en la Bahía Esperanza y en la Isla Decepción. Por fortuna, tales eventos no trascendieron a mayores (Ferrada Walker, 2015). Debido a los hechos ocurridos, el Reino Unido presentó demandas contra los países ante la Corte Internacional de Justicia en 1955 y exigió la invalidez de las reclamaciones de ambos (Ferrada Walker, 2015). Estados Unidos por su parte, desato controversia por el ejercicio militar highjump entre 1946 y 1947, desatando tensión especialmente con la Unión Soviética que, para esa época, ya había instalado bases de investigación (Ferrada Walker, 2015). Definitivamente, era hora de negociar entre los estados involucrados, con el fin de evitar futuros conflictos. Este espiral de sucesos sin duda dieron pie a la iniciativa del Tratado.

En 1959, laintención de buscarnuevosescenarios geoestratégicos era evidente e imperante para asegurar la obtención de posiciones dominantes ante el resurgimiento de cualquier conflicto de talla mundial. En plenos albores de la Guerra Fría, se llegó a un consenso histórico, tal vez de los más icónicos para el fortalecimiento de las relaciones internacionales entre los estados. La firma del Tratado Antártico se da como resultado del Año Geofísico Internacional que convocó a países como Argentina, Chile, Australia, Bélgica, Francia, Nueva Zelanda, Noruega, Sudáfrica, Estados Unidos y la -en ese entoncesparadigmática Unión Soviética. Estos países, reunidos en Washington, firmaron el Tratado con el objeto de resguardar el territorio antártico y destinarlo para las actividades científicas en beneficio de la humanidad (Instituto Antártico 
Chileno (INACH), 2019). El Tratado Antártico entró en vigor dos años después (1961), luego de que todos los países signatarios lo ratificaran desde sus respectivos parlamentos.

Desde la entrada en vigor del Tratado Antártico, se han establecido bases temporales que se activan sólo en el verano austral y permanentes, con un periodo de actividades del año completo, tiempo durante el cual se releva a las personas que allí permanecen (científicos y militares) en periplos de 365 días (COMNAP, 2917). Asimismo, se han llevado a cabo sendos estudios de bioprospección y diferentes investigaciones que involucran a las ciencias físico-químicas y a los factores medioambientales relacionados con el cambio climático, entre otros.

\section{COLOMBIA EN LA ANTÁRTICA: UNA APROXIMACIÓN POLÍTICO- ESTRATÉGICA}

Desde una postura critico-reflexiva, este escrito tiene como propósito vislumbrar algunos aspectos que sugieren la inclusión, en el proyecto de establecimiento de la base de investigación temporaria colombiana "Almirante Padilla" en la Antártica, otras aristas provenientes de la conceptualización transdisciplinaria que varios expertos voluntariamente han decido aportar. En primera instancia, se ha realizado un mapeo de ideas previas con el fin de encontrar aproximaciones conceptuales al tema en cuestión. A partir de esto, surgieron las siguientes proposiciones colectivas:

- La importancia científica, académica y social de desarrollar proyectos de ultramar para el Estado colombiano

- Reposicionar el concepto de la importancia de la base científica "Almirante Padilla" ante el actor decisorio de Estado
- $\quad$ Proponer la generación de anclajes transdisciplinarios

- Cooperación internacional - Bases conjuntas

- $\quad$ Retos en la era "pospandemia" para la financiación del Proyecto Antártica

- $\quad$ EI CONPES 3990 como oportunidad

- La concepción social del Programa Antártico Colombiano desde diferentes campos de acción estratégicos, tales como: Geopolítica, geoeconómica e investigación

- No es la Armada de Colombia en la Antártica, es el país en el continente blanco.

Luego de esbozar estas ideas, se plantea la siguiente pregunta de investigación que será abordada en el presente documento: ¿cuál ha sido la principal limitante para el avance del Programa Antártico Colombiano?

Valdría la pena dar una mirada a un enunciado 2001 que podría denominarse "justificación de país" y que fue base de motivación a inicios de la década de los 90 cuando, por una iniciativa del gobierno, se creó la Comisión Nacional para Asuntos Antárticos. Con respecto a la importancia de este continente para Colombia, en la exposición de motivos de la Ley 1880 de 2018, que aprobó el Protocolo al Tratado Antártico sobre Protección del Medio Ambiente, se ha afirmado lo siguiente:

Para el Gobierno de Colombia el continente Antártico representa un interés especial por varias razones, dentro de ellas, el papel que juega como regulador del clima mundial y como insuperable laboratorio para entender los fenómenos climáticos y sus efectos. Los países más desarrollados se encuentran realizando importantes investigaciones en variados campos y Colombia no puede seguir alejada de este estratégico centro mundial de producción 
científica. (Exposición de motivos Ley 1880, 2018)

Este postulado no ha perdido vigencia, pero es pertinente evaluar su resignación e incluir a otros proponentes como, por ejemplo, la Asociación Colombiana de Ciencias Políticas, con el objeto de dar la connotación complementaria a lo que podría denominarse "proyecto país".

Por otro lado, dentro de los criterios adoptados para la elaboración de este estudio, se acudió a una técnica de comunicación estructurada para la recolección de información: el método Delphi. Éste se basa en la consulta a un grupo de expertos a quienes se les plantean, de manera individual, una serie de preguntas para obtener una opinión fiable de consenso (Reguant-Álvarez \& Torrado-Fonseca, 2016). Durante la aplicación de este método, comúnmente se experimenta la necesidad de hacer varias rondas de interacción con las respuestas recopiladas en la primera comunicación para obtener una aproximación consensuada. Particularmente, en este ejercicio, el último proceso no fue necesario, teniendo en cuenta que las respuestas obtenidas reflejaban aspectos coincidentes desde las diferentes disciplinas consultadas.

Para el ejercicio académico, se contó con la ayuda de oficiales de la Armada de Colombia en actividad y de la reserva activa, especialistas en ciencias del mar, politólogos, internacionalistas, comunicadores, académicos y pedagogos. En total, se trabajó con 23 profesionales, además de los oficiales que han comandado las cuatro expediciones antárticas a bordo del buque de la Amada de Colombia ARC 20 de Julio².

2 "EI ARC 20 de Julio es una patrullera oceánica construida en los astilleros de COTECMAR en el año 2010 y puesta a disposición de la Armada Nacional el 5 de febrero de 2012, con capacidades para operaciones navales de patrullaje y vigilancia, búsqueda y rescate, así como interdicción marítima. Además, cumple con la dinámica, complejidad y versatilidad que le permite participar en operaciones de contención de derrame de hidrocarburos y también adecuar sus instalaciones para el desarrollo de estudios de investigación" (Bustos Luna, 2015). Ha sido la unidad de la Armada de Colombia con la que se han llevado a cabo las cuatro expediciones antárticas.
La intención era aproximarse a una reflexión propositiva mediante la exploración de saberes-otros, que involucran profesionales en otras disciplinas con conocimiento del Programa Antártico Colombiano (PAC). Una vez recopilada la información, se procedió a un análisis descriptivo tipo PESTEL, el cual busca identificar factores externos claves en un entono estratégico como instrumento de apoyo a la toma de decisiones, teniendo en cuenta determinantes políticos, económicos, sociales, tecnológicos y legales (Corvo). Esto permite la evaluación de un escenario particular en el presente para avizorar necesidades y cambios significativos en prospectiva.

El enfoque de este estudio se direcciona hacia las limitaciones político-estratégicas, normativas $y$ de recursos que pudiesen ser determinantes para materializar la intención del Estado colombiano de posicionarse en el continente antártico, hacer parte de los proyectos científicos de gran envergadura, y pertenecer al Tratado Antártico (TA) como miembro consultivo con voz $y$ voto en la toma de decisiones en el escenario geoestratégico. Las expediciones antárticas colombianas se adoptan como base para aproximarse a la materialización del Programa Antártico Colombiano.

\section{LAS CONSIDERACIONES POLÍTICO-ESTRATÉGICAS}

Desde el realismo clásico de Morgenthau, hasta el reflectivismo de la escuela de Frankfurt o el neoliberalismo de Hayek, se podría hallar una coincidencia en las teorías de las relaciones internacionales, respecto a la necesidad de un estado moderno de posicionarse en la Antártica. Como ya se ha mencionado anteriormente, no solo por la relevancia de los estados que allí han asentado sus bases temporales y permanentes desde la firma del Tratado Antártico, sino por lo que significa el Continente Blanco para 
el mundo en materia de recursos naturales, minerales y de bioprospección, sin duda punta de lanza para la elaboración de modelos predictivos que nos indiquen los cambios que, en materia climática, experimentará el mundo en un futuro próximo. Este enunciado da pie a la introducción de la primera parte que, a juicio de los expertos consultados, es determinante para la materialización con éxito del Programa Antártico Colombiano (PAC).

El Almirante Ernesto Duran González, ex comandante de la Armada de Colombia, quien fungió además como Director General Marítimo del país y fue un actor fundamental durante tres de las cuatro expediciones que se han adelantado a bordo de un buque de la Armada hacia la Antártica, manifestó lo siguiente:

En la primera expedición, la Armada Nacional rompió la inercia que permanecía desde 1990 cuando se despertó el interés antártico en Colombia. En 2014, una marina integral y con la capacidad de buques versátiles que se han construido en Colombia logró hacer el primer viaje con éxito, un suceso que marcó un hito en la historia del país y sus instituciones. Sin embargo, el escepticismo en términos políticos para la toma decisiones, en parte por la ausencia de conciencia marítima, fue una constante. Luego de sendos esfuerzos, se logró obtener la voluntad política, cuya consecuencia directa sería el desembolso de los recursos. Ello se da pese al freno del contra argumento sobre el porqué deberíamos ir a la Antártica sin antes haber resuelto asuntos más prioritarios como el litigio con Nicaragua. (E. Durán, entrevista, 23 de junio de 2020)

Este análisis es fundamental si se tiene en cuenta que un amplio sector político del país se ha familiarizado con la relevancia de los intereses marítimos como proyección de los intereses nacionales. Tal es el caso de la aceptación y puesta en marcha de la Política Nacional de los
Océanos y Espacios Costeros (PNOEC) que, desde el 2007, ha sido la carta de navegación que le permite al alto gobierno la promoción y direccionamiento de sus intereses marítimos. Sumado a esto, la PNOEC ha facilitado la interacción multisectorial en procura de la relevancia de la geografía marítima como parte constitutiva del Estado (Comisión Colombiana del Océano, 2018).

Sin embargo, es claro que, dentro de los 14 intereses marítimos nacionales que han sido contemplados en la PNOEC, sólo uno de ellos concuerda con las aspiraciones de Colombia en la Antártica, a saber, el número 5: "Investigación científica, tecnológica y de innovación" (Comisión Colombiana del Océano, 2018, p. 52). Aun así, la PNOEC plantea el manifiesto interés del país de posicionarse en el continente austral y de justificar las relaciones directas entre los procesos de conservación y protección ambiental que el Estado ha desarrollado y que son congruentes con el texto del Tratado Antártico. Por medio de la PNOEC también se busca un enfoque que lleve a comprender los impactos antrópicos en temas tan relevantes para la humanidad y el país como el cambio climático (Comisión Colombiana del Océano, 2018).

El Vicealmirante Orlando Romero Reyes, segundo comandante de la Armada de Colombia, manifestó, respecto a la materialización del proyecto de la base de investigación "Almirante Padilla" y las expediciones a la Antártida, lo siguiente:

Ya estamos en la Antártica, ya somos visibles ante el mundo en este escenario. Ahora es necesario que esta experiencia adquirida en las expediciones ya realizadas se catapulte e inserte el tema antártico dentro del ADN del Estado colombiano. Con esto quiero decir que, a partir de esa convicción de Estado de ser parte consultiva del Tratado Antártico -que ya está 
haciendo carrera y va muy bien-, se defina un plan para lograr los pasos necesarios para tener una base compartida o individual en la Antártica. (O. Reyes, entrevista, 10 de julio del 2020)

De acuerdo con Luis Alexander Montero, politólogo, magister en análisis de problemas políticos y candidato a PhD en estudios políticos, una gran falencia es que el mar no está presente, de manera tácita, en la definición de interés nacional, lo que da cuenta de la poca atención que recibe en la Antártica. A nivel de otras instituciones del Estado, tampoco existe un sentimiento de oportunidad, lo que ha llevado a que sólo la Armada de Colombia participe en las actividades relacionadas con la Antártida, a pesar de que es una responsabilidad compleja en la que debe haber participación multisectorial. Montero (entrevista, 16 de julio de 2020) resume en dos aspectos cruciales su postura: la ausencia de interés nacional y la precariedad en la voluntad política.

Es importante resaltar las posiciones desde diferentes disciplinas y observar aproximaciones en cuanto a la necesidad indemne de la voluntad política. Al respecto, es claro que el Estado colombiano cuenta con las herramientas jurídicas y constitucionales que le permitirían un diseño estratégico hacia la Antártica. Lo anterior desde un camino que ya ha sido ampliamente explorado por científicos, universidades colombianas y, por supuesto, por la Armada de Colombia. Sobre este aspecto, el Capitán de navío (RA) Samuel Ignacio Rivera, PhD en ciencias sociales y humanas, hace referencia a la ausencia de una cultura estratégica como un elemento que debería ser adoptado para el direccionamiento de la política exterior colombiana. El desarrollo de la investigación y las expediciones antárticas han sido una iniciativa de la Armada de Colombia, antes que un esfuerzo de todo el país. Esto está ligado a una visión estratégica menguada que, de una $\mathrm{u}$ otra manera, es inducida históricamente por la tendencia de la clase dirigente a una mirada etnocentrista de Estado, que limita la manera de ver el mundo estratégicamente. Además, temas como la Antártica o iniciativas como la Alianza Pacifico, entre otras, son necesarias si Colombia busca posicionarse en el mundo geoestratégicamente como actor relevante ( $\mathrm{S}$. Rivera, entrevista, 2 de agosto de 2020).

En este punto, el profesor Henry Cancelado Franco, politólogo de la Universidad Nacional de Colombia y magister en conflictos contemporáneos, tiene una posición similar, ya que enfatiza en la "visión internista" del nivel político y la reticencia del país para lanzarse a la investigación profunda por una timidez científica que persiste. Todo esto induce la generación de temores en el diseño de proyectos de gran envergadura como lo requeriría el posicionamiento de Colombia en la Antártica ( $\mathrm{H}$. Cancelado, entrevista, 10 de julio de 2020). Además, Cancelado (entrevista, 10 de julio de 2020) insiste en que la proyección de la política exterior de un estado juega un papel preponderante en la legitimación y consolidación ante el sistema y la sociedad internacional.

Por otro lado, Ana María Aragón Holguín, PhD en ciencias de la educación y experta en educación superior en Colombia, expresa la necesidad de materializar el Programa Antártico Colombiano con la presencia de varias instituciones en el Continente Blanco (entrevista, 23 de junio de 2020). Aragón Holguín hace énfasis en la importancia de conceptualizar este proyecto desde las ciencias humanas y, desde la ethopolitica, comprometer a los gobernantes mediante un dialogo que paralelamente involucre a la sociedad, a las universidades públicas y privadas, y a los ministerios de defensa, educación, hacienda y ciencia, tecnología e innovación (A. Aragón, entrevista, 23 de junio de 2020).

Aragón (entrevista, 23 de junio de 2020) también manifiesta que, cuanto más amplia sea la cobertura en materia participativa para 
los temas antárticos, la fluidez en la toma de decisiones para la asignación de recursos daría pie a la materialización del proyecto. Este último debe ser planteado como un concepto que haga referencia a lo que se quiere transmitir desde una aproximación de proyecto de país (A. Aragón, entrevista, 23 de junio de 2020. Además, Aragón explica que es necesario fundar una nueva triada como un escenario de mayor representación que tenga tres ejes: educación, desarrollo e investigación. Esto permitiría avanzar en la configuración de una auto/eco/posición de país sobre el tema, del cual se desprenda una Política Nacional Antártica (A. Aragón, entrevista, 23 de junio de 2020).

Andrés Franco Herrera, PhD en oceanografía, experto en ciencias del mar y expedicionario antártico colombiano, explica sobre la necesidad de crear una cultura bioceánica en el país, cuya base está en la educación (A. Herrera, entrevista, 26 de mayo de 2020). Además, Herrera aclara que no se trata de cambiar contendidos programáticos, sino de redireccionar la manera de enseñar. Según Herrera, si Colombia no logra entender la importancia de la Antártica para el mundo, no existirá un interés genuino del geoposicionamiento estratégico en el Continente Blanco (entrevista, 26 de mayo de 2020). Para Franco Herrera, la expedición antártica y el posicionamiento de la base Almirante Padilla en ese continente debe convocar instituciones educativas, empresas privadas y al Estado, pero sobre todo a la sociedad. En este proceso, se debe instar la idea de posicionarse en la Antártida con base en aproximaciones conceptuales desde la geopolítica, la geoeconómica, la seguridad alimentaria, la biodiversidad y el cambio climático (A. Herrera, entrevista, 26 de mayo de 2020).

Es preciso hacer énfasis en los dos últimos postulados y destacar la participación de expertos con un alto sentido de pertenencia frente a la institucionalidad quienes, desde sus disciplinas, podrían aportar con una particular transferencia de lo inter a trans. Esta condición los aleja del sesgo profesional y los llevaría a coincidir en la relevancia de la interacción de la comunidad académica, investigativa, científica y del Estado. Una integración de esta naturaleza derivaría en la generación de una conciencia colectiva respecto a la Antártica y en la necesidad de hacer parte de ese selecto grupo de países que cuentan con bases de investigación destacadas.

Según el Capitán de Navío (RA) Sergio Uribe Cáceres, $\mathrm{PhD}$ en derecho internacional y docente e investigador de la Escuela Superior de Guerra Rafael Reyes Prieto, el Programa Antártico Colombiano debe ser visto como un proyecto de Estado y debe ser congruente con los intereses de la nación. Además, el Programa necesita involucrar a los diferentes actores que pudiesen enarbolar su relevancia, tales como instituciones educativas, la empresa privada, medios de comunicación, entre otros. Uribe Cáceres también afirma que es necesaria la participación de los diferentes ministerios y la integración de la Armada de Colombia como un medio para la consecución de una finalidad de Estado (entrevista, 26 de mayo de 2020).

En otras secciones de este documento, se plasmarán algunos postulados de otros expertos que compartieron sus aproximaciones a este trabajo académico.

\section{EL PROGRAMA ANTÁRTICO COLOMBIANO Y SUS DOCUMENTOS RECTORES: LO NORMATIVO}

En cuanto la participación de Colombia y su presencia activa en la Antártica se han logrado avances significativos en los últimos años, logros que se han materializado en el desarrollo del Programa Antártico Colombiano y en el planeamiento y ejecución de las investigaciones científicas a bordo de buques o estaciones pertenecientes a países aliados. También, estos 
progresos se han visibilizado en el desarrollo de las expediciones lanzadas con fuerza propia con unidades de la Armada de Colombia y de la Fuerza Aérea Colombiana.

Ante el escenario planteado anteriormente, se deben considerar las aspiraciones de poner en funcionamiento una estación científica durante el verano austral que, posteriormente, podría ser permanente, así como los campamentos requeridos con miras a lograr la llegada al Polo Sur Geográfico. Todo esto hace necesario establecer qué tanto soporte existe desde el punto de vista legal y documental para materializar este interés estratégico.

Es pertinente mencionar que, en la actualidad, el gobierno del presidente Iván Duque cuenta con el Plan Nacional de Desarrollo 2018-2022 en el cual, de acuerdo con los objetivos de Desarrollo Sostenible de las Naciones Unidas y con el propósito de construir equidad verdadera y sostenible, se plantean veinte metas que buscan transformar a Colombia. Entre otros, se destaca el Pacto por la Ciencia, la Tecnología y la Innovación, el cual se pensó como un sistema para construir el conocimiento de la Colombia del futuro. Este último se define como "el conocimiento científico y desarrollo de tecnología e innovaciones para transformar la sociedad colombiana y su sector productivo" (DNP - Departamento de Planeación Nacional, 2019).

Llama la atención que, pese al interés manifestado por el gobierno en apoyar el Programa Antártico Colombiano (PAC), además de que el Pacto por la Ciencia, la Tecnología y la Innovación se encuentra orientado a aumentar la inversión pública y privada, en ningún aparte del Plan Nacional de Desarrollo se hace alusión al sostenimiento del interés científico en la Antártica y tampoco hace referencia al aumento de la presencia nacional en dicho territorio. $\mathrm{Si}$ se sigue la trazabilidad de los documentos rectores que soportan la participación en el
Tratado Antártico, se encuentra la Política de Defensa y Seguridad -derivada del Plan Nacional de Desarrollo- la cual, dentro de sus objetivos estratégicos y líneas de política, en el numeral 6.1.4., hace alusión a la preservación y defensa del agua, la biodiversidad y los recursos naturales como activos estratégicos de la Nación y de interés nacional. También, se menciona el fortalecimiento del Programa Antártico con el fin de preservar los intereses estratégicos de la Nación (Ministerio de Defensa Nacional, 2019). Con esto, se evidencia el interés del sector defensa por apoyar el sostenimiento y crecimiento de la investigación científica y la presencia activa en la Antártica como un activo de relevancia estratégica y geopolítica.

En lo que refiere al marco legal, se encuentra respaldo de la Ley 1880 de 2018 por medio de la cual se aprobó el Protocolo al Tratado Antártico sobre Protección del Medio Ambiente, su Apéndice y sus Anexos. Esta ley, en su preámbulo, menciona que los estados parte del Protocolo son conscientes de la necesidad de incrementar la protección del medio ambiente antártico y sus ecosistemas dependientes y asociados. Por lo anterior, las partes se comprometieron a la protección global del medio ambiente antártico y designaron a la Antártica como una reserva natural cuyos fines específicos sean la paz y la ciencia. Para este fin, las partes deberían cooperar en la planificación y realización de actividades en el área (Ley $1880,2018)$. Uno de los factores más relevantes era prohibir la intervención en el ecosistema mediante cualquier actividad relacionada con recursos minerales. Los anexos del Protocolo al Tratado Antártico sobre Protección del Medio Ambiente tienen contenido relevante para las partes. Por ejemplo, el anexo II hace referencia a la conservación de la flora y la fauna presentes en estos ecosistemas, y el anexo IV busca la prevención de la contaminación marina mediante el manejo de residuos. 
Otro documento relevante en este recuento es el CONPES 3990, cuyo título - "Colombia Potencia Bioceánica Sostenible 2030"- da cuenta de un gran plan diseñado en prospectiva y de connotación estratégica, busca el posicionamiento de los océanos como un factor de desarrollo sostenible ante la agenda pública nacional. Sin duda, este CONPES representa una oportunidad para la nación y ha sido un salto estratégico para la generación de una cultura colectiva bioceánica. Como parte del diagnóstico en el ámbito científico, se describe el contexto de las expediciones científicas a la Antártica en los siguientes términos:

En el 2014, en el marco del Comité Técnico Nacional de Asuntos Antárticos (CTN-AA), se creó el Programa Antártico Colombiano (PAC) con el fin de promover la investigación científica nacional en el escenario internacional de la Antártica (CCO, 2020). Sin embargo, el PAC no ofrece lineamientos de política pública articulados y financieramente sostenibles. De hecho, la financiación es asumida fundamentalmente por la Armada Nacional y la DIMAR, quienes han aportado más del $90 \%$ del presupuesto en 4 de las 6 expediciones científicas realizadas a la fecha, lo que suma cerca de 7.500 millones de pesos por expedición. Esto resulta en bajos niveles de autogestión de las expediciones científicas de Colombia a la Antártica, al recaer la financiación en la Armada Nacional y DIMAR. (DNP - Departamento Nacional de Planeación, 2020, p. 38)

Pese al desalentador diagnóstico, las expectativas pueden ser focalizadas en el CONPES 3990 y la visión 2030 que contempla:

Colombia reconoce como parte de su identidad nacional sus zonas costeras, marinas e insulares en el Pacífico y en el Caribe, así como su ubicación estratégica entre el Pacífico y el Atlántico, su vecindad con el Canal de Panamá, su participación en alta mar y la Antártica. (DNP
- Departamento Nacional de Planeación, 2020, p. 59)

Este escenario es prometedor, toda vez que, a partir de este objetivo, se espera contar con una mayor participación en aras de fortalecer la proyección como potencia bioceánica, así como una mayor participación en escenarios internacionales relacionados con los océanos y los intereses marítimos.

Igualmente, el CONPES 3990, a través de la línea de acción 3.3., busca impulsar las expediciones científicas nacionales e internacionales con la finalidad de fortalecer las capacidades científicas y técnicas para el conocimiento sobre los océanos y la protección de los intereses marítimos nacionales. Para ello, el Departamento Administrativo de la Presidencia de la Republica consolidará el Programa Nacional de Expediciones Científicas como una medida interinstitucional a largo plazo, además de impulsar las expediciones como estrategias interdisciplinarias e interinstitucionales para profundizar en el conocimiento $y$ aprovechamiento de los recursos marinocosteros. En esto, se incluyen convocatorias de líneas de investigación relacionadas con el conocimiento, aprovechamiento y bioprospección.

Adicionalmente, y a través de la Comisión Colombiana del Océano, se elaborará una propuesta de procedimientos para la planeación, alistamiento y ejecución de las expediciones científicas a la Antártica, lo que permitirá generar espacios intersectoriales e interinstitucionales para la consolidación del Plan Nacional de Expediciones Antárticas (DNP - Departamento Nacional de Planeación, 2020). Lo anterior refleja un firme interés antártico en la agenda política colombiana.

Por su parte, el Programa Antártico Colombiano actúacomounahojaderutaquebuscamaterializar los intereses nacionales en la Antártica, así 
como el desarrollo de las directrices que lleven a Colombia a tener un papel más influyente y dinámico en las decisiones del Tratado Antártico. El objetivo general del Programa es "posicionar al país como un actor preponderante dentro del Sistema del Tratado Antártico, incidiendo en la toma de decisiones globales sobre ese territorio" (Comisión Colombiana del Océano, 2015, p. 17). No obstante, se debe precisar que, mientras el país no cuente con políticas de Estado que asignen responsabilidades sin regulación por periodos de gobierno, estas incitativas no se materializarán para beneficio de la nación.

Colombia también cuenta con los lineamientos estipulados en la Agenda Científica Antártica 2014 -2035, la cual funge como un documento orientador donde se formulan las estrategias en el desarrollo de las actividades científicas de Colombia en el Continente Blanco. Además, la Agenda estipula el desarrollo de la investigación que se inició en el 2014 con proyecciones hasta el año 2035 y contempla los lineamientos para que entidades y universidades realicen investigación continua en la Antártica, bien sea con plataformas propias, plataformas o bases de otros países o desde el campo teórico. Todo esto con el propósito de que Colombia genere conocimiento para aportar a la solución de problemas actuales y futuros, y realizar contribuciones al Sistema del Tratado Antártico (Comisión Colombiana del Océano - Comité Técnico Nacional de Asuntos Antárticos, 2014).

Finalmente, vale la pena mencionar la Política Nacional de los Océanos y los Espacios Costeros, que se ha tomado como la carta de navegación que le permite al país direccionar y promover sus intereses marítimos, y generar un espacio para reconocer las distintas acciones de todos los sectores del Gobierno y de la sociedad. Todo esto, en procura del reconocimiento del mar, las costas y las regiones insulares como parte constitutiva del Estado colombiano. La Política Nacional de los Océanos y los Espacios
Costeros, en su versión 2016 -3030, proyecta el derrotero de Colombia hacia una potencia media oceánica, a través de lineamientos de cooperación e integración en asuntos marinos, desarrollo económico, aprovechamiento sostenible de los recursos, ordenamiento del territorio marino-costero, protección de la biodiversidad y la defensa de la soberanía. En la ejecución de estos lineamientos siempre se procura el bienestar de los colombianos (Comisión Colombiana del Océano, 2020) y se contemplan los asuntos antárticos y la importancia que este tema representa para el Estado colombiano.

Entre otros apartados, la Política Nacional del Océano y los Espacios Costeros considera que es primordial el establecimiento de sinergias globales en pro de una presencia activa en escenarios internacionales que involucren los asuntos del mar (participación en organismos internacionales afines, desarrollo de investigación en la Antártica, apoyo a misiones de seguridad global, fortalecimiento de mecanismos regionales sobre el mar, entre otros) (Comisión Colombiana del Océano, 2018).

Así las cosas, se puede aducir que Colombia no carece de documentos rectores en cuanto a normatividad se refiere para el avance del Programa Antártico. Sin embargo, es pertinente analizar el alcance de estos y el impacto que generan en la toma de decisiones, por parte del ejecutivo, sobre los recursos necesarios para la materialización de las proyecciones ya documentadas en los instrumentos mencionados. Al respecto, el Contralmirante Juan Francisco Herrera Leal, Director General Marítimo de Colombia, postula que existen razones claras y concretas para entender la relevancia del diseño del marco legal para asegurar una presencia permanente que genere un concepto de posesión o posición en la Antártica (J. Herrera, entrevista, 17 de junio 
de 2020). Entre las múltiples razones que benefician a Colombia, se encuentra su posición del meridiano que permite la proyección de la Isla Malpelo. Según Herrera Leal, si se crea un marco legal sería un acto de soberanía y de posición que nos otorgaría un privilegio hacia los demás estados (J. Herrera, entrevista, 17 de junio de 2020).

Andrés Usuga Marín, oficial de la reserva de la Armada de Colombia, académico y especialista en derecho constitucional y derecho del mar y marítimo, expresa la falencia que existe desde la interpretación de la Constitución Política de Colombia que, en su Artículo 101, indica lo siguiente:

También son parte de Colombia, el subsuelo, el mar territorial, la zona contigua, la plataforma continental, la zona económica exclusiva, el espacio aéreo, el segmento de la órbita geoestacionaria, el espectro electromagnético y el espacio donde actúa, de conformidad con el derecho internacional o con las leyes colombianas a falta de normas internacionales. (Const., 1991, Art. 101)

Según Usuga, con respecto a la Antártica, es necesario un debate de discusión pública en la academia y los medios de comunicación, con el soporte jurídico que otorga la Constitución (A. Usuga, entrevista, 2 de agosto de 2020). Así mismo, hace énfasis en lo determinante que es para Colombia apropiarse, en todo sentido, de este artículo constitucional en pro de la defensa de los intereses nacionales, no solo en el litigio con Nicaragua, sino para reposicionar el concepto del interés antártico y su importancia geoestratégica para el país (A. Usuga, entrevista, 2 de agosto de 2020).

\section{CONSIDERACIONES FINALES}

El principal reto del Estado colombiano es identificar los intereses que lo motivan para pertenecer al Tratado Antártico, una posibilidad que obligaría a movilizar la capacidad nacional para asumir los compromisos y tareas que se derivan de este propósito nacional. La evolución ha sido lenta desde que Colombia manifestó el deseo de pertenecer al Tratado Antártico que, en principio, se aleja de las pretensiones y los reclamos de soberanía. La Armada de Colombia, junto con otras entidades, asumió el reto de reiniciar las expediciones para revivir ese interés nacional. Este nuevo impulso solo se logró hasta el 2020 en el momento en que se oficializó el depósito del Instrumento de Adhesión al Protocolo de Protección Ambiental del Tratado Antártico, ante el Departamento de Estado de los Estados Unidos, país depositario del Tratado.

El Protocolo de Protección Ambiental del Tratado Antártico o Protocolo de Madrid se firmó el 4 de octubre de 1991 y está vigente desde 1998. Es decir, han transcurrido 20 años de discusiones para que Colombia oficialice su interés y compromiso. La protección ambiental es primordial para el Sistema del Tratado Antártico y el Protocolo funciona como marco de gobernanza en el continente blanco que se conoce como una "reserva natural consagrada a la paz y la ciencia", según lo manifestado por la Presidencia de la República en el comunicado oficial del depósito.

Así las cosas, ante la comunidad internacional, Colombia ha dado grandes pasos para convertirse en miembro consultivo del Tratado Antártico, lo que le permitirá participar con voz y voto en la toma de decisiones sobre la conservación, preservación y administración de este continente. Además, le permitirá reconocer el gran impacto global de esta región para el país como uno de los principales reguladores del clima y de los océanos, condición sine qua 
non para continuar en la carrera de exploración permanente a través de una base científica en la Antártida.

Los caminos que quedan incluyen establecer alianzas con países que ya tienen bases en la Antártida en un programa de cooperación que sea viable en temas presupuestales y logísticos. También, se deben sostener las expediciones permanentes cada año alineadas a los objetivos estratégicos nacionales y del Tratado, y convencernos, como nación, de que el destino de nuestro país está ligado a lo que suceda en el último rincón del planeta que podemos y debemos conservar.

Es importante tener en cuenta que la Antártida no ha sido una parte fundamental de la agenda nacional. Desde los años ochenta, el asunto lo puso sobre la mesa la Armada Nacional en conjunto con otras entidades, y lo extrapoló a las esferas políticas. Sin embargo, no es un asunto de interés nacional por muchas razones. A pesar de las circunstancias, no se descuida el tema y, con los años, se toma la decisión de alistar un buque por encima de cualquier circunstancia y se llega por primera vez a la Antártida en buque propio, construido en Colombia y tripulado por marinos colombianos.

Lo anterior demuestra la importancia del paso a paso en un proyecto de esta envergadura. Por lo tanto, tener una base en la Antártica reviste complejidad y más cuando a ello se suma las circunstancias actuales de recesión económica por la pandemia que azota a la humanidad. Sin embargo, un trabajo continuado interinstitucional debe continuar para fortalecer el programa como "proyecto país" que permita esa materialización desde el compromiso inclusivo de todo el estamento estatal. Este proyecto debe ser liderado por una cabeza ministerial que ejerza como actor primario y que genere directrices y recursos, además de apoyar a un ente integrador como la Comisión Colombiana de los Océanos.
Las Fuerzas Militares de Colombia juegan un papel fundamental y serán los llamados a proyectar el poder del Estado en otras latitudes, además de funcionar como un componente logístico por excelencia. También, es necesario alcanzar un estándar donde los ingenieros militares del Ejército Nacional asuman un papel en el Programa Antártico y la Fuerza Área Colombiana continúe con sus capacidades y habilidades de aterrizaje en el continente Blanco. Finalmente, la Armada de Colombia deberá liderar ese gran componente de desplazamiento y sostenibilidad.

Por último, se deben mejorar las comunicaciones estratégicas que permitan el despliegue informativo de lo que significa la Antártica para el país. Esta acción se debe extrapolar a la educación primaria y secundaria, dado que en estos escenarios se gesta la conciencia bioceánica, ligada al concepto de territorialidad, desarrollo y soberanía. Ángela Posada Swafford, periodista científica y expedicionaria antártica, manifiesta que "[e]l día que, a cada barrio de Colombia, a cada escuela, y a cada municipio, llegue la información sobre la importancia de la Antártica para el país, ese día se hará realidad el proyecto en todas sus dimensiones".

NOTA: Agradecimientos especiales a los profesionales que hicieron posible el desarrollo de este trabajo académico. Su aporte representa una evolución en el pensamiento estratégico colombiano e insta a la consolidación de $s$ redes de interacción académica interdisciplinaria, de las cuales siempre será necesario depender para aprender.

- $\quad$ Almirante (RA) Ernesto Durán, Ex Comandante Armada de Colombia

- Vicealmirante Orlando Romero, Segundo Comandante Armada de Colombia

- Vicealmirante Antonio Martínez, Comandante Fuerza Naval del Pacífico 
- Vicealmirante (RA) Juan Manuel Soltau, Ex Secretario ejecutivo CCO

- $\quad$ Contralmirante juan Francisco herrera, Director General Marítimo de Colombia

- $\quad$ Capitán de Navío William Pedroza, Jefatura intereses marítimos y relaciones internacionales de la Armada de Colombia

- Capitán de Navío Juan Forero, secretario ejecutivo CCO

- $\quad$ Capitán de Navío Joaquín Urrego, excomandante ARC 20 de julio

- $\quad$ Capitán de Navío Fernando Diaz, excomandante ARC 20 de julio

- $\quad$ Capitán de Navío Adelmo Dam Tous, expedicionario antártico

- Capitán de Navío Camilo Segovia, excomandante ARC 20 de julio

- $\quad$ Capitán de Navío (RA) Sergio Uribe, Docente Investigador Escuela Superior de Guerra, PhD en Derecho Internacional Marítimo.

- $\quad$ Capitán de Navío (RA) Samuel Ignacio Rivera, Docente Investigador Escuela Superior de Guerra, PhD en Ciencias Sociales y Humanas

- Capitán de Navío (RA) Hernando Osorio, Docente Investigador Escuela Superior De Guerra

- $\quad$ Capitán de Navío (RA) Rafael Ricardo Torres, PhD en Oceanografía

- $\quad$ Capitán de Navío (RA) Jorge Espinel, Docente Investigador Escuela Superior De Guerra

- $\quad$ Capitán de Corbeta Wilson Ríos

- $\quad$ Capitán de Corbeta Rafael Hurtado

- Doctor Henry Cancelado, Docente Investigador Escuela Superior De Guerra,
Politólogo de la UN, Magister en análisis de problemas contemporáneos.

- Doctora Angela Swafford, periodista científica, autora y editora colombiana

- Doctor Andrés Franco, Biólogo marino de la Universidad Jorge Tadeo Lozano PhD en oceanografía

- Doctora Ana María Aragón PhD en Ciencias de la Educación

- Doctor Alexander Montero, Politólogo (c) PhD Ciencias Políticas

- Doctor Andrés Úsuga, Abogado especialista en derecho constitucional y derecho del mar

\section{REFERENCIAS BIBLIOGRÁFICAS}

Alberini, C. (1971). Historia del pensamiento filosófico argentino. Argentina.

Armadaespañola.(2019).LaAntártida. Continente helado. Obtenido de Armada española: https:// armada.defensa.gob.es/ArmadaPortal/page/ Portal/ArmadaEspannola/conocenosespeciales/ prefLang-es/06aniversarios--09xx-annoscampanna-antartica--02tratado

Astigarraga, E. (2015). El método delphi. Universidad de Deusto - Facultad de CC.EE. y Empresariales.

Bordese, F. G. (2008). Develando el continente antártico. Ediciones para Estudios Politécnicos de América.

Bustos Luna, G. (2015). Especial ICEMAN: Preparación ARC 20 de Julio. Obtenido de Centro de Investigaciones Oceanográficas e Hidrográficas-ClOH: https://www.cioh.org. co/index.php/es/2015-07-27-19-20-22/1725especial-iceman-preparacion-arc-20-de-julio. html 
Campaña Científica ANTAR XXVII. (Marzo de 2020). Bitácora. Obtenido de Campaña Científica ANTAR XXVII Perú 2019-2020: https://www.dhn. mil.pe/antar27/index.php?seccion=bitacora

Cancelado, H. (10 de julio de 2020). Limitantes Politicas, Logisticas y Normativas para el establecimeinto de Colombia en la Antàrtica. (M. Lopera, Entrevistador) Bogotà, Cundinamarca, Colombia.

Centro cultural argentino de montaña. (2009). Historia del sector Antártico Argentino. Revista digital de montaña(19). Obtenido de Centro cultural argentino de montañ: http://www. culturademontania.org.ar/Historia/HIS_historiaantartida-argentina.htm

Céspedes, J. A. (15 de enero de 2020). 026 Literatura peruana - Fichas (436-454) - Pedro Sancho De la Hoz. Recuperado el 30 de julio de 2020, de Editorialdelosandes.over-blog. com: http://editorialdelosandes.over-blog. com/2020/01/026-literatura-peruana-fichas-436454-pedro-sancho-de-la-hoz.html

Comisión Colombiana del Océano - Comité Técnico Nacional de Asuntos Antárticos. (2014). Agenda Cientifica Antártica de Colombia 2014 - 2035. Comisión Colombiana del Océano. Obtenido de http://www.cco.gov.co/docs/ publicaciones/Agenda_Antartica.pdf

Comisión Colombiana del Océano. (2015). Programa Antártico Colombiano. Comisión Colombiana del Océano. Obtenido de http://www. cco.gov.co/docs/publicaciones/p_antartico.pdf

Comisión Colombiana del Océano. (2018). Política Nacional del Océano y de los Espacios Costeros. Comisión Colombiana del Océano. Obtenido de http://www.cco.gov.co/cco/ publicaciones/83-publicaciones/383-politicanacional-del-oceano-y-los-espacios-costerospnoec.html
Comisión Colombiana del Océano. (2020). Comisión Colombiana del Océano. Obtenido de Publicaciones Comisión Colombiana del Océano: http://www.cco.gov.co/cco/publicaciones/83publicaciones/383-politica-nacional-del-oceanoy-los-espacios-costeros-pnoec.html

Comisión para la Conservación de los Recursos Vivos Marinos Antárticos. (23 de octubre de 2019). Convención de la CRVMA. Obtenido de Comisión para la Conservación de los Recursos Vivos Marinos Antárticos: https://www.ccamlr. org/es/organisation/convención-de-la-crvma

COMNAP. (2917). Antartic Station Catalogue. Council of Managers of National Antartic Programs COMNAP.

Congreso de la Republica de Colombia. (2018). Ley 1880 de 2018 "Protocolo al Tratado Antártico sobre Protección del Medio Ambiente, su Apéndice y sus Anexos I, II, III y IV". Bogotá D.C.

Corin, C. (28 de enero de 2018). Efemérides: 2020, de Tus buenas noticias-Argentina: https:// tusbuenasnoticiasarg.blogspot.com/2018/01/ efemerides-28-de-enero.html

Corvo, H. S. (s.f.). Análisis PESTEL: para qué sirve, factores, cómo hacerlo, ejemplo. Obtenido de Lifeder.com: https://www.lifeder.com/analisispestel/

DNP - Departamento de Planeación Nacional. (2019). Plan Nacional de Desarrollo 2018 2022. Obtenido de https://colaboracion.dnp.gov. co/CDT/Prensa/PND-2018-2022.pdf

DNP - Departamento Nacional de Planeación. (2020). Colombia Potencia Bioceánica Sostenible 2030 Documento CONPES 3990. Bogota D.C.

Escobar, J. d. (2020). Campaña Científica Antar XXVII. Marina de Guerra de Perú. 
Estirado, L. (14 de diciembre de 2016). Primera expedición al Polo Sur: ¿Por qué Amundsen llegó antes? El Periódico. Obtenido de El Periódico: https://www.elperiodico.com/es/ extra/20161214/primera-expedicion-al-polo-surroald-amundsen-5689122

Europa Press. (15 de septiembre de 2017). ¿A quién pertenece la Antártida? Obtenido de Europa Press: https://www.europapress. es/internacional/noticia-quien-perteneceantartida-20170915152034.html

Ferrada Walker, L. V. (2015). La Antártica ante la Corte Internacional de Justicia: A 60 años de los casos Reino Unido c. Chile y Reino Unido c. Argentina. Revista Tribuna Internacional, 4(7), 155-172. doi:10.5354/0719-482X.2015.36984

Gabarró i Raurich, X. (2013). Expediciones marítimas a la Antártida, aspectos metereológicos, oceanográficos, glaciológicos y de la navegación entre hielos. Tesis de pregrado, Universidad de Cantabria, repositorio institucional. Obtenido de https://repositorio. unican.es/xmlui/handle/10902/4054

Gavaldà, J. (11 de junio de 2019). Jacques Cousteau, el gran defensor de los mares y océanos. Obtenido de National GeographicHistoria: https://historia.nationalgeographic. com.es/a/jacques-cousteau-gran-defensormares-y-oceanos_14353/1

GeoEnciclopedia. (s.f.). Océano Ártico. Recuperado el 30 de julio de 2020, de GeoEnciclopedia: https://www.geoenciclopedia. com/oceano-antartico/

Green Peace. (2020). Preguntas y respuestas sobre la Antártida. Obtenido de Green Peace: https://es.greenpeace.org/es/preguntasfrecuentes/preguntas-y-respuestas-sobre-laantartida/

Green Peace Chile. (2020). Protege la Antártica. Obtenido de Green Peace Chile: https://www. greenpeace.org/chile/involucrate/protege-laantartica/

Instituto Antártico Chileno (INACH). (9 de mayo de 2019). Sistema del Tratado Antártico. Obtenido de Instituto Antártico Chileno (INACH): https://www.inach.cl/inach/?page_id=21

Ministerio de Defensa Nacional. (2019). Politica de Defensa y Seguridad. Ministerio de Defensa Nacional. Ministerio de Defensa Nacional. Obtenido de https://www.mindefensa.gov. co/irj/go/km/docs/Mindefensa/Documentos/ descargas/Prensa/Documentos/politica_ defensa_deguridad2019.pdf

Pinedo, E. (s.f.). 7 datos sorprendentes sobre la Antártida. Recuperado el 30 de julio de 2020, de National Geographic: https://www.ngenespanol. com/ciencia/7-datos-sorprendentes-sobre-laantartida/

Real Academia Española. (2005). Antártida. En Diccionario Panhispánico de Dudas. Real Academia Española. Recuperado el 1 de agosto de 2020, de https://www.rae.es/dpd/ Ant $\%$ C3\%A1rtida

Reguant-Álvarez, M., \& Torrado-Fonseca , M. (2016). El método Delphi. Revista d'Innovació $i$ Recerca en Educació, 9(1), 87-102. doi:10.1344/ reire2016.9.1916

Rowlatt, J. (29 de enero de 2020). Derretimiento de la Antártica: por qué los científicos advierten que un glaciar del tamaño de Reino Unido es "el más peligroso del mundo". Obtenido de BBC News: https://www.bbc.com/mundo/ noticias-51281366

Talarn, J. (6 de marzo de 2017). La Antártida, un termostato que regula el clima del planeta. Obtenido de Agencia EFE: https://www. efe.com/efe/america/cronicas/la-antartidaun-termostato-que-regula-el-clima-delplaneta/50000490-3198908 